to the other members of the family, no member getting the same response. These responses were predetermined by the $E$ from previous frequencies of correct and incorrect responses on this type of problem. All families were given the same sequence. At no time does any member of the family have access to any information from another member.

It was predicted that the consensus-sensitive families would perform (1) poorly on the public mode, in which they would have maximal conflicting, shared evidence, (2) fair on the anonymous mode, and (3) best on the standard mode.
Environment-sensitive families should perform equally well in all modes. The predicted direction is illustrated in Figure 1. Analysis of the pre- and posttest inventories tended to confirm the hypothesis.

To conclude, I would like to read three observations made by Dr. Reiss as major advantages over noncomputer methods:

(1) Observer rating scales, long bedeviled by problems of reliability and validity, can be eliminated since all of the family's performance is scored automatically.

(2) The experimental procedure is precisely standardized and can be exactly replicated using a copy of our program tape and the same standard Teletypes and computer equipment.

(3) The presence of the human $E$ has been partially eliminated, and the prospect is good for his total unemployment.

We hope to soon eliminate the human element in this procedure, but, as it stands, it severely strains a $2 \mathrm{~K}$ memory, even with microtapes. We expect to get about 3 years' good usage out of these procedures before transferring to an anticipated larger computer system.

\section{DECUS DISCUSSION}

DR. GOFF: Can you comment on new software for the PDP-12?

RICHARD CLAYTON: The most significant initial aspects of the software of the 12 is a new operating system including assembler, editor, and various peripheral programs to handle filing and program conversion, etc. It is currently dubbed the DIAL system, or Display Interacting Assembly Language. The program is patterned in the interactive sense, very much after the LAP-6 program, which Mary Allen Wilkes distributed some 2 years ago, and the user characteristics, at least in an editing sense, are very similar. The assembly characteristics are slanted very much toward the PDP-12. Specifically, it is an assembler that handles either of the two order codes, and the format for assembly, and henceforth the format with which one writes source programs. The format for the assembler is very similar to the one commonly used throughout the DEC machines. Specifically, it has six character alphanumerics plus slash for the comment delimiter, large character $i$ as the indirect bit, and different symbol characters. This departure from the classic LINC format we felt obligated to go to in trying to bring the two machines together in a single assembly language that would allow rapid, convenient assembly of programs in either or both modes. In detail, the program allows, within core, some 180 user-defined symbols, outside of the instructions that are part of the symbol set. Beyond 180 symbols, the tape is used as the symbol area, yielding, of course, a substantial decrease in the assembly rate. As soon as one goes to mass storage for the symbol table (even if one does very clever things for symbol searches-if you have to go out to some mass storage in the form of LINC tapes or DECtape), the assembly slows down fast. To that end, an additional $4 \mathrm{~K}$ of core or disk will be usable for the symbol table. The $8 \mathrm{~K}$ version is in the program but not running at the moment. We will be running DIAL at Spring Joint for those of you who are there. The machine that is there is, in effect, a production-type machine. There will be workable versions of DIAL with the first PDP-12s that go out, which are still a couple of weeks away. That will be an evolving program, and I think we'll see quite a few changes to that in the form of additions in the next year and a half. [NOTE: The name DIAL has been changed to LAP 6-DIAL because of the similarity of many of the operating features of the editor portion of the system to those of LAP.6. Although the present name is LAP 6-DIAL, the responsibility for all development, corrections, and distribution will rest with Digital Equipment Corporation.]

SEVERO ORNSTEIN: Dick, I think that one part of the laboratory instrument meaning of the LINC was the terminal frame. What have you done to it [data terminal panel assembly]?

RICHARD CLAYTON: It existed in the form of two large plug-in units in the classic LINC. In the case of LINC-8, it consisted of about 50 unused slots mounted behind the door in the upper right-hand side of the front. What happened to that on the PDP-12? To some degree, it has gone "bye-bye," a little bit anyway. We have put a large number of options prewired into the machine itself. So a number of some of the things that went into the data terminal panel assembly, such as plotters, additional Teletypes, certain things with display and $A$ to $D$, have been prewired within the machine. This provides economy of manufacturing and allows the user to request of the manufacturer that these be supplied. Further, it makes it practical to supply them at a reasonable cost and with a minimum of labor for installation and expansion of the machine. For other kinds of options, available literally "off the shelf," there are, for example, IBM-compatible tape, things of this sort These are also offered as standard options and plug onto an $\mathrm{I} / \mathrm{O}$ bus.

The data terminal panel concept, as originally implemented in the classic LINC, allowed up to two boxes to be plugged into the machine and used some predecoded operate lines. What one ran into was a limitation in the total number of device codes, if you like, available for easy interfacing. The interface structure was extremely simple but was not a bus structure. We would now call it a radial structure in that it had several sets of ports into the machine. Once those were used up in the straightforward way, you were all over, and that was in general the way one implemented the first interfaces one designed. Beyond that, one had to make a bus structure out of one of these radial ports, so that several devices could be put 
into the $\mathrm{SN}$ and $\mathrm{TN}$ lines of the classic LINC. This meant that at some point, the interface had to be redesigned if one wanted many interfaces. A number of classic LINCs have gone that route. The basic concept of the PDP-8, LINC-8, or PDP.12 I/O bus is that of a LINC $\mathrm{I} / \mathrm{O}$ bus structure which is independent of the number of devices connected to it. The cables for that interface connection go from device to device. In this case, a device consists of a mounting panel of logic with or without its own power. As one adds or takes off devices, they have any 1 of 64 device codes which provides you potentially with up to 64 devices, and the machine structure becomes rather independent of what combination of peripherals are on the machine. It is not quite as convenient to plug and unplug these devices as it would the data terminal panels of the classic LINC. But the independence in intermixing of options is much more practical, as in the implementation of these in a production sense-specifically, the ability to buy these options independent of what you currently have and our ability as a manufacturer to supply them independent of the current status of your machine. And it is with that philosophy that we do not have the mounting panels per se.

With the LINC-8, a number of people put nice little interfaces in the data terminal panel, for one or two things, but then, if they wanted to make an elaborate inferface, they went to separate mounting panels anyway, and the door and panel were rather cumbersome. Now the plug-in option becomes even more cumbersome and more expensive in many cases. There is still some room inside the machine itself, and the mounting panels for creating this kind of an interface are readily available from our standard catalog of logic parts; the interface is again described as a bus-type interface.

DR. STROEBEL: I would like to ask Mary Allen Wilkes a question. Possibly one of the great successes of the LINC concept today has been the software which has been written by bright users in the field. Is there any way you can see of encouraging this flow of software so that new application programs become more readily available? You have certainly been concerned about this for a long time; for example, your Washington University group, under the direction of Wes Clark, maintains a bibliography of all papers published as scientific literature which involve some kind of LINC processing. Do you have any thoughts on this?

M. A. WILKES: Well, I think one thing that we've learned is that it is not easy to keep the program flow, as you refer to it, going successfully between users. I should perhaps clarify one thing-when we started the bibliography that you referred to, we were not primarily interested in acting as a program-information exchange group. DECUS does this already, and I still encourage people to submit programs to DECUS. We were interested in keeping track of the documented research articles that described finished work, or work in progress, that would be of interest to other scientists in the same field, or to scientists working in different fields, but who are interested in using computers in the same way. This has been valuable because most of the journal articles listed on the bibliography do not, in fact, mention the computer itself at all, and that's fine. That means it has properly been integrated into the laboratory and is being used as a research tool. But it is very difficult for new LINC users to track the information down without a bibliography.

As to the program-flow business, I'm sure someone from DECUS is better able to answer to this. We do not attempt to exchange programs at all between people. If they see something on the bibliography that looks as though it is something they can use, then fine. They are encouraged to write to the author or, if it is available through DECUS, to write to DECUS. We cannot maintain tapes or other people's documentation.

I would certainly say this about distributing programs. And that is, there is no point in trying to distribute a program which has nothing written down about it. And there are a number of people who write programs who seem to think that because a program is useful to them, and they know how it works because they wrote it, or the guy who sits next to them wrote it, it is a tremendous benefit to someone in California. The documentation has to be plain, it has to be complete, and it has to be such that someone can run the program on his own, by using only the information he can get through the mail. So, if you want to exchange programs, I would first encourage this kind of attitude toward documenting them.

DR. STROEBEL: Would publication of programs in some more formal kind of scientific publication to permit legitimate publication credit encourage this kind of documentation?

M. A. WILKES: I'm not sure. I think the programs go with the instrument itself, with the computer as part of the tool. The two work together to hopefully do some useful work. If there are programming techniques that are learned in writing the program, these are perhaps properly documented in some of the computer journals. I don't think that the detailed workings of a program should be documented in the scientific journals, although you see it occasionally. I don't think this is too useful. A lot of teaching of some of the "higher-level languages," for example, is based on information that is available only in journal articles, and this information is only descriptive of the language. It does not attempt to tell you exactly how to use the language or to write programs in the language, and these descriptions are almost impossible to use as programming guides. So, from that standpoint, I wouldn't encourage it, not when it encourages an author to be just a bit more general than he should be about the way the program, or the language, actually works.

S. ORNSTEIN: I have a comment. I can't let Dick [Richard Clayton] get away quite as easily as he did when he was talking about the data terminal panels, because I think that one of the things that we're seeing here is the standardization that we very carefully built out of the first machine. And I see it creeping in, and I don't like it very well, because the whole theory underlying our data terminal box was that we didn't know what we were going to connect to. It was put out there specifically to make it easy, not for the manufacturer of the machine, but for the user who wanted to use it for his special clear kind of thing.

1 understand that it's easier now to get the parts to plug in so that we can interface to this system or the IBM tape system, and that's fine if that's what half of them want to do. But I think that this whole basic concept was concerned with putting a computational device in the laboratory with a whole lot of other apparatus that we didn't understand. So, to make it easy for people to adapt the LINC to their own unique requirements, we left the data terminal as flexible as possible. I would also like to ask really why you did choose to eliminate it? Was it just packaging convenience? Why did you choose to put the machine all into one piece as you have? I have heard people say that's not quite so handy. Would you like to answer that?

R. CLAYTON: Before I do, I understand Severo's concerns here on the data terminal panel question, and they are very valid. I think I indicated our attempt there is to go to a format where readily available components may be used to make up whatever type of interface is appropriate for the special equipment within the laboratory-and on the context 
of the I/O bus, with readily available cables, and panels to do it from. We also don't feel that we can specify how big that is, and that unless we force everybody to buy a long, large one, we then get into other types of limiting problems. In addition, I would like to make one point. We have proceeded quite a ways in the last 6 years to a point where a number of users are very interested in the machine as a tool and much less interested in the interfaces per se, at least for a large number of peripherals, many of which are quite common. And that's what leads to the desirability of having a number of peripherals readily available at low cost. On the question of packaging, there are some very obvious economies involved in not interconnecting machines with elaborate cable structures. The cable structure of the classic LINC was a rather expensive one and added quite a bit to the manufacturing costs and to some of the subtleties and difficulties of the check-out and operation of the machine. We also felt that it imposed very severe restrictions on moving the machine.

DR. STROEBEL: Dr. Hiltz has agreed to share some of his experiences on the use of process-control computers in neurophysiology applications.

DR. HILTZ: Thank you. As active users of small computers in real-time biological work, you might find some interest in similar applications of the larger breed of real-time computers usually known as process-control machines. Not long after 1 wrote the DATAVG on-line signal-averaging program for the LINC-8, I went to Cornell, where an IBM 1800 had recently been installed in a laboratory for radiation biology and neurophysiology. My first interest there was to write a similar signal-averaging program for the 1800 . A comparison of the two programs is interesting in itself and should bring out some more general differences between the two classes of computer. Perhaps a word about the process-control computer per se is in order first.

These machines look rather attractive for real-time lab work. They are designed to run subroutines in response to "outside" events rather than under direct control of an "inside" program. To this end, they make heavy use of a priority interrupt structure and $\mathrm{I} / \mathrm{O}$ devices which run on data channels, that is, independent of program control. Analog and digital input and output are readily available.

However, the design of these computers to control processes such as steel production or oil refining leads to a philosophy that is not particularly attractive in the research laboratory. I speak of the 1800 now, but it is typical of the process-control computers. The idea of protection is important in process-control work. This shows up in two important ways. First, the several "users" of the computer will include different parts of the controlled process as well as programmers debugging new routines, and perhaps something like accounting going on in the background. All these users must be assumed to run completely independently of one another and therefore must be completely protected from one another. This is accomplished by a sophisticated monitor, or executive system, which assigns sections of core storage and different $\mathrm{I} / \mathrm{O}$ devices among the users and prevents each from using what belongs to the others. The monitor also provides graceful recovery from errors by the users. All these goodies naturally require a lot of monitor, in the case of our 1800 , about 6,500 words out of the 16,000 available.

The second aspect of protection is protection of the process. Once the computer is in control, it had better stay in control, or else we are likely to find ladles of steel running off the ends of their tracks, etc. Thus, for every interrupt, there must be a service subroutine. Of course, we use a dummy subroutine for all these in our lab and attach our working programs only to the interrupts which they use. This is fine for the first attempt at a program, which is, of course, full of bugs. When we have a new version, we can't simply delete the old one and put in the new. As far as the monitor is concerned, that would leave some part of the process uncovered for a time, so it won't permit deletion of a real-time program. We must instead replace the old version with a dummy, then compile the new version and replace the dummy with the new version. This takes only a small handful of control cards. Protection of this sort is just not applicable in the lab environment; we have spent a great deal of time working around the many ways in which the system protects the user both from himself and from others, of which the above is just one example.

Let's return to the signal-averaging programs as examples of how the two kinds of computers can handle a lab problem. The LINC-8 program DATAVG runs a loop which displays the accumulating sum of signals while waiting for a trigger pulse on a sense line. Then it samples an analog input voltage, which places the value in the accumulator. From there, it is added to the accumulating sum in the core, which is then scaled and displayed right from the accumulator. Simple. Direct. All this action except the
Table 1

A Comparison of the LINC- 8 and IBM 1800

LINC-8 $\quad \underline{1800}$

Core for storage

of accumulating

sums

$2048 \quad 2560$

Maximum number

of analog input

channels

8

16

Maximum input

rate, samples per

second

Live display

$11000 \quad 8000$

Display of a

reference signal

waveform Yes No

Display after

adjustable display

format

Inhibition of

averaging on

contingencies

Computation of

standard deviation

of the mean

Yes Yes

Yes No

Yes No

Back ave raging

(signal precedes

trigger)

Yes $\quad \mathrm{No}^{*}$

* Standard deviation computation later added to 1800 program.

display may be inhibited by the occurrence of any of several contingencies, made known via analog inputs or sense lines, so (for example), only responses evoked during positive EEG voltages will be included in the average.

The 1800 program is, of necessity, somewhat more complex. The trigger signal activates an interrupt. The monitor executes 169 instructions in delivering control to my program from that trigger pulse. Naturally, we can't afford to wait that long, so I have taken over the hardware interrupts. These must remain in core, however, to handle interrupts from the system I/O devices, such as the disk, which I don't care to program myself. The trigger interrupt service routine starts the $A \cdot D$ converter. Because the converter operates on a data channel independent of programs, it, of course, cannot put its sample value in the accumulator. Rather, it leaves the value in a table previously established in core and sets a flag when done. The program waits for this flag, during which time it can't do much else, then brings the analog value from core into the accumulator, from which it is added to the accumulating sum. Our CRT display operates on another data channel, taking its data from a table in core. For display, the program would have to scale the 
accumulating sum, set some bits which give microcoded commands to the display and deposit the result in the display table. It turned out that there was just not enough core for yet another table, so the live display was dropped. Inhibition of averaging based on external contingencies was discarded as too awkward and time-consuming to be worthwhile, basically because the program runs so automatically.

In short, for this type of program at least, the LINC-8 runs rings around the 1800. See the comparison in Table 1 .

B oth programs use a keyboard-interactive set-up routine, and both make extensive use of program overlays from mass storage.

In answer to the question about the future of the LINC-8 program DATAVG, I have been asking people about whether this should be improved for the PDP-12. I don't know yet whether we should work out a PDP. 12 version. The only real differences would be to take advantage of the faster A-D converter and the built-in clock. The LINC-8 version requires an external clock or some sort of pulse generator in the laboratory to connect to one of the sense lines.

QUESTION: Are there any positive things you can say about the 1800 as a typical process-control computer?

DR. HILTZ: Yes. It's nice to have all that core, and it's nice to have FORTRAN. Even in our on-line lab work, at least half of our programming is of the sort that can be done very comfortably in FORTRAN. The inefficiency of assembler coding is, of course, the amount of effort between the perception of the problem and a workable solution in the computer. Here, FORTRAN is much better. I would want a PDP-12 with 8K of core so I can run such programs in FORTRAN, and I would hope that the assembler in DIAL would offer the ability to link subroutines with FORTRAN calling programs.

DR. STROEBEL: Most LINC-8 users may not realize that a carefully documented procedure is already available to combine LAP programs with FORTRAN routines which are processed by the PDP-8 part of the computer. The procedure, called "LINC-TRAN" has already been published as Program L-21 in the DECUS Library. The general strategy used by LINC-TRAN on the LINC-8 should be considerably simplified for the PDP-12. 